\title{
Does Anticipation of Pain Affect Cortical Nociceptive Systems?
}

\author{
Carlo A. Porro, ${ }^{1}$ Patrizia Baraldi, ${ }^{2}$ Giuseppe Pagnoni, ${ }^{2}$ Marco Serafini, ${ }^{4}$ Patrizia Facchin, ${ }^{1}$ Marta Maieron, ${ }^{1}$ \\ and Paolo Nichelli ${ }^{3}$
}

1Dipartimento di Scienze e Tecnologie Biomediche, Università di Udine, I-33100 Udine, Italy, Dipartimento di 2Scienze Biomediche and 3Patologia Neuropsicosensoriale, Università di Modena e Reggio Emilia, I-41100 Modena, Italy, and ${ }^{4}$ Azienda Sanitazia Locale Modena, I-41100 Modena, Italy

Anticipation of pain is a complex state that may influence the perception of subsequent noxious stimuli. We used functional magnetic resonance imaging (fMRI) to study changes of activity of cortical nociceptive networks in healthy volunteers while they expected the somatosensory stimulation of one foot, which might be painful (subcutaneous injection of ascorbic acid) or not. Subjects had no previous experience of the noxious stimulus.

Mean $\mathrm{fMRI}$ signal intensity increased over baseline values during anticipation and during actual stimulation in the putative foot representation area of the contralateral primary somatosensory cortex (SI). Mean fMRI signals decreased during anticipation in other portions of the contralateral and ipsilateral SI, as well as in the anteroventral cingulate cortex.

The activity of cortical clusters whose signal time courses showed positive or negative correlations with the individual psychophysical pain intensity curve was also significantly af-

Functional imaging studies in experimental animals and in humans have disclosed a distributed cortical network related to pain (Porro and Cavazzuti, 1996; Casey, 1999; Ingvar and Hsieh, 1999; Treede et al., 1999, 2000). There is evidence for a physiological role of frontal, insular, cingulate, and parietal regions in perceptual aspects, such as intensity coding (Derbyshire et al., 1997; Porro et al., 1998; Coghill et al., 1999). The crucial role of the cerebral cortex in pain mechanisms is highlighted by positron emission tomography (PET) investigations (Rainville et al., 1997; Bushnell et al., 1999; Petrovic et al., 2000; Hofbauer et al., 2001), showing that pain-related activity of the anterior cingulate and of the primary somatosensory cortex (SI) may be changed by hypnotic suggestions or attentional modulation influencing affective or sensory components of pain, respectively. Understanding mechanisms underlying cognitive modulation of the pain system is a crucial challenge, on both theoretical and clinical grounds (Price, 1999).

Anticipation of pain is a complex state that may influence the immediate unpleasantness of pain (Staub et al., 1971; Price, 1999)

\footnotetext{
Received Aug. 27, 2001; revised Jan. 9, 2002; accepted Jan. 23, 2002.

This work was supported by grants from the Ministry of Research and Consiglio Nazionale delle Ricerche (Italy) to C.A.P., the University of Modena and Reggio Emilia to P.B., and the Azienda Policlinico (Modena, Italy) to P.N. We thank C. Murari, F. Lui, and E. Dassi for their help in data analysis.

Correspondence should be addressed to Prof. Carlo A. Porro, Dipartimento di Scienze e Tecnologie Biomediche, Università di Udine, Piazzale Kolbe 4, I-33100 Udine, Italy. E-mail: cporro@makek.dstb.uniud.it.

G. Pagnoni's present address: Department of Psychiatry and Behavioral Sciences, Emory University, Atlanta, GA 30322.

Copyright (C) 2002 Society for Neuroscience $\quad 0270-6474 / 02 / 223206-09 \$ 15.00 / 0$
}

fected during the waiting period. Positively correlated clusters were found in the contralateral SI and bilaterally in the anterior cingulate, anterior insula, and medial prefrontal cortex. Negatively correlated clusters were found in the anteroventral cingulate bilaterally. In all of these areas, changes during anticipation were of the same sign as those observed during pain but less intense $(\sim 30-40 \%$ as large as peak changes during actual noxious stimulation).

These results provide evidence for top-down mechanisms, triggered by anticipation, modulating cortical systems involved in sensory and affective components of pain even in the absence of actual noxious input and suggest that the activity of cortical nociceptive networks may be directly influenced by cognitive factors.

Key words: anticipation of pain; pain perception; primary somatosensory cortex; cingulate cortex; insular cortex; medial prefrontal cortex; functional magnetic resonance imaging

and of non-noxious stimulation (Sawamoto et al., 2000). It is likely to involve several factors, such as cognitive appraisal, arousal, conditioning, and orienting or diverging attention from the source and site of noxious input; the importance of these factors may vary according to the instructions given to the subject and to past experience (Hsieh et al., 1999). Hemodynamic changes in parietal, cingulate, and insular areas, but not in the somatotopically appropriate portion of SI, have been detected by PET during anticipation of painful stimuli (Drevets et al., 1995; Chua et al., 1999; Hsieh et al., 1999). Two recent functional magnetic resonance imaging (fMRI) studies have investigated anticipation-related changes in cortical activity: one study suggested a separate system in the cingulate and insular cortex underlying the anticipatory state, distinct from the one involved during pain (Ploghaus et al., 1999), whereas the other demonstrated modulation of the activity of clusters responding to innocuous thermal input in the anterior cingulate cortex and parietal operculum/posterior insula (Sawamoto et al., 2000). Because pain perception may be influenced by anticipation, the activity of cortical nociceptive systems could be affected as well. This, however, has not been demonstrated so far.

We described previously, using fMRI, cortical clusters encoding pain intensity over time on the mesial hemispheric wall contralateral to noxious stimulation of one foot (Porro et al., 1998). In that study, we incidentally noted changes of activity after the warning signal but preceding stimulation onset. To further address this issue, we compare here the activity of cortical structures of the two hemispheres during anticipation of a poten- 
tially noxious stimulation and during actual somatic input. We aimed at investigating whether functional activity levels of regions involved in sensory and affective components of pain would be simultaneously affected during anticipation and whether the effect could be accounted for by arousal or by top-down (e.g., attentional) mechanisms. A specific goal was to discover possible spatial overlaps between anticipation- and pain-related changes of activity in the human cortex.

\section{MATERIALS AND METHODS}

We studied 30 volunteers after informed consent and approval of the Ethics Committee of the University of Modena and Reggio Emilia. Four of them were discarded from the analysis because of large movement artifacts. Therefore, we present data from 26 subjects.

\section{Experiment 1}

Twenty-one healthy right-handed volunteers (seven males and 14 females; age range of 19-31 years; mean of 25.1 years) were included in the study. The experimental protocol was similar to that described previously (Porro et al., 1998). Subjects were randomly assigned to one of two groups, regardless of gender: ascorbic acid $(n=14$; five males and nine females) or control ( $n=7$; two males and five females). Each participant was studied only once and was subjected to only one of two kinds of stimulation on the dorsum of one foot: subcutaneous injection of an ascorbic acid solution $(0.5 \mathrm{ml}, 20 \%)$ using a 23 gauge needle (ascorbic acid) or innocuous touching of the skin with a needle for $15 \mathrm{sec}$ (control). Subcutaneous injection of ascorbic acid induces, according to its concentration, moderate to strong burning pain lasting several minutes (Rossi and Decchi, 1997). The side of stimulation was balanced across individuals. Each experiment lasted $\sim 17 \mathrm{~min}$. A brief "warning" signal (cleaning the skin of the foot with an antiseptic solution for $10 \mathrm{sec}$ ) was delivered at minute 3 and was followed, 1 min later, by the stimulus (see Fig. 1, top). Heart rate was continuously monitored in all subjects.

Subjects were aware of which foot would be stimulated, of the time interval between the warning signal and the stimulus, and that the stimulation could be painful. However, they did not know in advance which stimulus would actually occur. None had ever experienced a subcutaneous ascorbic acid injection before. They were instructed to code the sensory intensity of perceived pain on a $0-100$ scale, in which 0 denoted "no pain" and 100 denoted "the maximum imaginable intensity of pain." It was explained that the estimate concerned the sensory intensity of pain and not unpleasantness. No hint was given on the expected intensity and duration of pain. Subjects were asked to communicate by conventional signs, using both hands, pain intensity at $1 \mathrm{~min}$ intervals during the whole course of the experiments (both before and after stimulation) in response to a light touch of the unstimulated foot. Specifically, they were instructed to lift their hands and fingers twice in close succession, the first time to indicate the tens (1-10 lifted fingers was $10-100)$ and the second one to indicate the units. Closed hands meant 0. The whole motor sequence could usually completed in 3-4 sec. Apart from this, they were asked to refrain from moving any part of the body throughout the experimental period. Subjects had their eyes open during the scanning.

Data acquisition and analysis. Functional images were acquired over the entire experimental period from 15 contiguous axial planes, using a 1.5 T GE Horizon Hispeed $77 \mathrm{MR}$ system and a $\mathrm{T}_{2}{ }^{*}$-weighted gradientecho echo-planar sequence (nihepi; courtesy of P. Jezzard, FMRIB Center, John Radcliffe Hospital, Oxford, UK) [repetition time (TR) of $15 \mathrm{sec}$; echo time $(\mathrm{TE})$ of $48 \mathrm{msec}$; $64 \times 64$ matrix; pixel size, $3.75 \times$ $3.75 \times 6 \mathrm{~mm}$, interpolated to $2 \times 2 \times 2 \mathrm{~mm}$; 68 volumes per run]. Spin-echo $\mathrm{T}_{1}$-weighted axial images from the same planes (TR of 500 msec; TE of 9 msec; $256 \times 256 ; 0.94 \times 0.94 \times 6 \mathrm{~mm})$ and whole-head spoiled gradient-recalled acquisition in a steady state $\mathrm{T}_{1}$-weighted images (TR of $35 \mathrm{msec}$; TE of $9 \mathrm{msec}$; 124 slices; $0.94 \times 0.94 \times 1.3 \mathrm{~mm}$ ) were also acquired to be used as anatomical references for regional identification and transformations into the Talairach space (Talairach and Tournoux, 1988), respectively. Volumes were later aligned using the AFNI package (Cox, 1996).

We performed two different kinds of analyses: the first one on mean fMRI signal changes in identified regions of interest (ROIs) (see below) and the second one on the fMRI signal changes in specific clusters related to perceptual aspects of pain, identified through correlation analysis. By the first approach, one can quantitatively analyze the overall behavior of an anatomically identified area, without any a priori assumption on the kind of response and without the need to apply arbitrary statistical thresholds. Because the results of brain imaging techniques, including fMRI (Logothetis et al., 2001), appear to be attributable mainly to synaptic activity, we thus obtained a picture of the overall afferent input in specific cortical regions during the waiting phase and after stimulation. By the second approach, we aimed to examine signal changes during the anticipatory phase in well characterized nociceptive clusters.

Regions of interest included different portions of the postcentral gyrus and of the cingulate cortex, medial prefrontal cortex, and insula in the two hemispheres. Their boundaries were outlined onto the individual structural images according to anatomical landmarks (Ono et al., 1990; Yousry et al., 1997), such as the pattern of cortical sulci and the position relative to the anterior and posterior commissures, and then projected onto the aligned functional images. The foot representation area of SI was defined as the portion of the paracentral lobule located posterior to the medial root of the central sulcus, which was identifiable in all subjects. The SI hand representation area was assumed as the portion of the postcentral gyrus corresponding to the precentral knob (Rumeau et al., 1994; Yousry et al., 1997), and the SI face representation area was assumed as the adjacent lateral portion of the postcentral gyrus, above the parietal operculum (see Fig. 2, top). Mean Talairach coordinates of the centers of mass of the identified regions (see legend of Fig. 2) were checked compared with those of activated foci during somatosensory stimulation of the foot, hand (Andersson et al., 1997; Gelnar et al., 1999), and face (G. Cruccu, personal communication). The cingulate cortex was divided into three portions: anteroventral, anterior, and posterior, following Devinsky et al. (1995). The insula was divided into two portions (anterior and posterior, including the parietal operculum) according to Greenspan et al. (1999). It is underlined that, given the interindividual variability in the cytoarchitectural and functional parcellation of cortical areas relative to sulcal anatomy (Rademacher et al., 1993; Vogt et al., 1995; Geyer et al., 1999) and in the functional localization of different body areas in SI, the identification of areal boundaries must be necessarily viewed as approximate, and terms such as the foot, hand, or face area of SI are used for descriptive purposes only.

The individual psychophysical pain profiles over the entire experimental period, adjusted for hemodynamic delay effects, were used as reference waveforms to identify clusters displaying signal changes related to pain intensity over time (Porro et al., 1998), thus creating statistical maps based on the correlation coefficient $r$ (Bandettini et al., 1993). We avoided spatial normalization of functional images before the analysis. This was done to identify even small clusters with greater anatomical detail. A value of $r=0.60$ and a cluster size of 10 pixels in the interpolated volumes were assumed as significance thresholds. This yielded an overall significance level of $<0.001$, corrected for the number of comparisons, as estimated by a Monte Carlo simulation of the process of image generation, spatial correlation of voxels, voxel intensity thresholding, and cluster identification (routine by B. D. Ward, Biophysics Research Institute, Medical College of Wisconsin, Milwaukee, WI). The resulting maps were transformed into the Talairach space using AFNI.

To allow interindividual comparisons of data acquired at different times during the experiment, fMRI signals were normalized in every subject and for each pixel by dividing the actual value of signal intensity in each image by the mean intensity value of the same pixel in the first eight images acquired during the initial period. Mean values from ROIs, or from identified clusters within each ROI, were then calculated and compared for three different epochs: baseline (corresponding to the first $2 \mathrm{~min}$ of the experimental period), waiting (corresponding to the $45 \mathrm{sec}$ immediately preceding stimulation), and poststimulus. The poststimulus epoch was defined as the first minute after stimulation when comparing mean values from the whole ROIs, including data from all of the 21 subjects (ascorbic acid plus control groups), and as the first 3 min after stimulation when comparing fMRI signals in the cortical clusters identified by correlation analysis (11 of 14 subjects from the ascorbic acid group; see Results). This was done because preliminary analyses showed that mean fMRI signal changes in ROIs, particularly after non-noxious stimulation, were short lasting. On the other hand, peak pain intensity (and hence peak fMRI signal changes in the identified clusters) occurred at different times (up to $3 \mathrm{~min}$; see Results) after the subcutaneous injection in different subjects of the ascorbic acid group.

fMRI data were analyzed by ANOVA, with group (ascorbic acid or control) as the between-subjects and side (contralateral or ipsilateral), region, and time (baseline, waiting, or poststimulus) as the withinsubjects factors. Student's $t$ test was used for post hoc comparisons if 


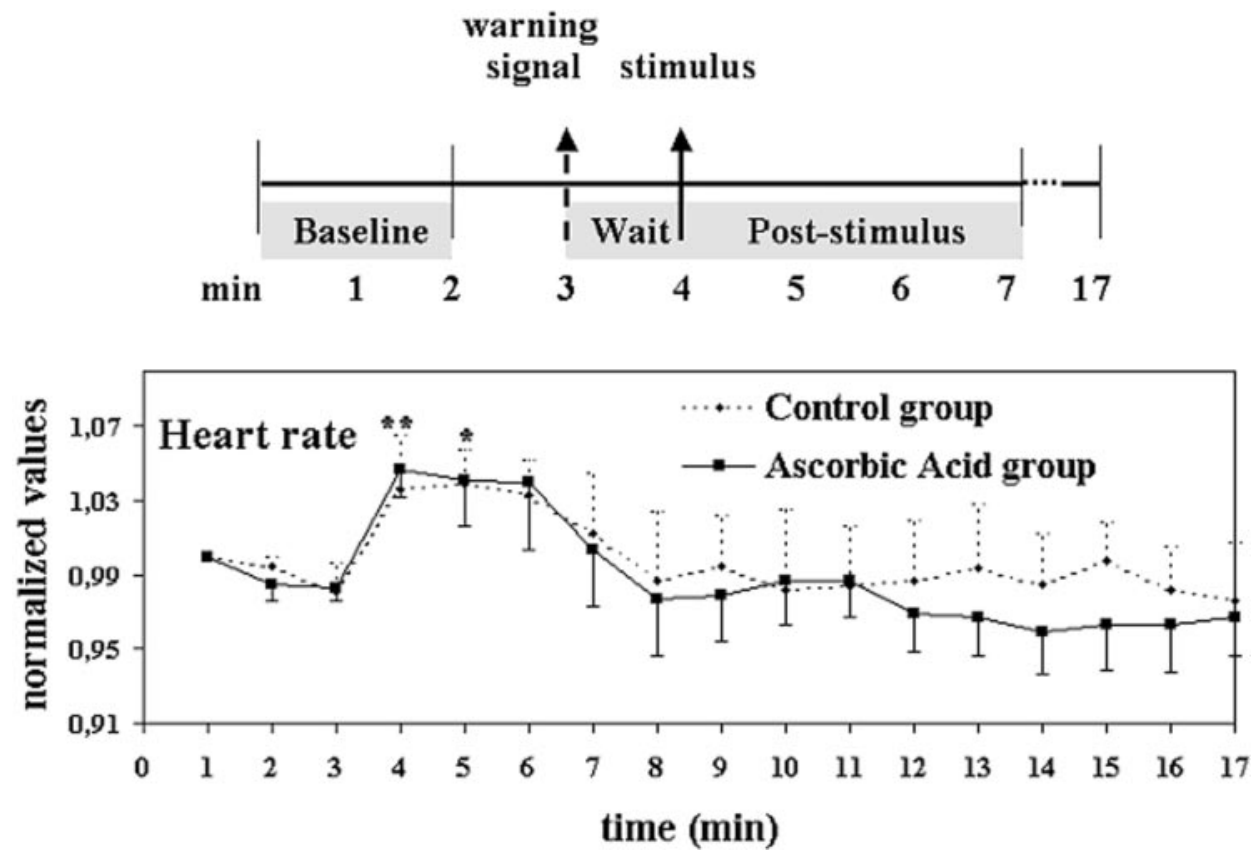

Figure 1. Experiment 1. Experimental dethe control and ascorbic acid groups during the experimental period. ${ }^{*} p<0.05$ and $* * p<0.01$ indicate values from the two groups significantly different from baseline, respectively. sign and mean + SEM heart rate profiles of

heart rate increase over baseline during the waiting period (from $76 \pm 3$ to $80 \pm 3$ beats $/ \mathrm{min}$; paired $t=3.67 ; p<0.01)$. No difference was found between the heart rate profiles of the control and ascorbic acid groups (ANOVA; group, $F=0.28$, NS; group $\times$ time, $F=0.43$, NS) (Fig. 1$)$.

All subjects of the ascorbic acid group experienced burning and aching pain after the subcutaneous injection. Peak pain intensity occurred from 1 to $3 \mathrm{~min}$ after the injection and showed large interindividual variations (range of 10-85; mean of $42 \pm 6$ ). Pain duration was also highly variable among individuals (range of 5-12 min; mean of $6 \pm 1$ ). No subject of the control group reported pain or discomfort. onto the skin horizontally for $5 \mathrm{sec}$ ) was delivered at the dorsum of the left foot at minute 3; subjects knew in advance that this was not followed by any additional stimulation. In the other two runs (cue condition), subjects were informed that the same tactile stimulus was to be followed 1 min later by a second stimulus, which could be either innocuous or noxious (subcutaneous injection of saline or ascorbic acid). In fact, only innocuous stimuli were applied to avoid sensitization. Run order was balanced across subjects. Functional images were acquired using the same equipment and fMRI sequence as in experiment 1 but with shorter TR ( $4 \mathrm{sec}$ ), thus yielding 75 volumes per run. Volumes were later aligned and Talairach-transformed using AFNI.

Data analysis was similar to the one described for experiment 1 . Boundaries of three regions of interest within the postcentral gyrus (approximately corresponding to the foot, hand, and face representation areas of SI) were outlined for each hemisphere onto the individual structural images and then projected onto the aligned functional images. Mean normalized fMRI signal intensity during the baseline and waiting epochs was compared by ANOVA with time (baseline or waiting), condition (control or cue), and side (contralateral or ipsilateral to the stimulated foot) as the within-subjects factors. To account for the different timing of acquisition from experiment 1 , the waiting period was defined as the $44 \mathrm{sec}$ immediately preceding stimulation onset in the cue condition or as the corresponding period in the control condition (including 11 functional volumes).

\section{RESULTS}

\section{Experiment 1}

In an open interview at the end of the experimental session, all subjects reported an enhanced attentional state and arousal after the warning cue attributable to anticipation of a potentially painful stimulus. This was paralleled by a moderate but significant

\section{Mean fMRI signal changes in selected regions of interest}

We first compared mean activity levels of the putative foot, hand, and face representation areas of SI during anticipated and real somatosensory stimulation of one foot. ANOVA of data from the hemisphere contralateral to the stimulated side showed that time had a different effect according to region (time, $F=3.24, p<$ 0.05 ; region, $F=5.46, p<0.01$; region $\times$ time, $F=3.86, p<$ $0.01)$. ANOVAs of data from individual regions revealed a significant effect of time in the foot representation area of SI $(F=$ 5.00, $p<0.02$ ), values acquired during the waiting period being higher than baseline (difference contrast, $t=2.24, p<0.05$ ). No significant effect was found in the ipsilateral hemisphere (Fig. 2). An additional analysis, based on a priori assumptions, on data from the hand and face areas of SI in the contralateral and ipsilateral hemispheres showed that, in these regions, values were lower than baseline during the waiting period (ANOVA plus difference contrast, $t=-2.35, p<0.05$ ). Signal increases in the contralateral foot SI were therefore selective, which argues against nonspecific arousal effects.

In the anteroventral cingulate, values from both the waiting (time, $F=5.84, p<0.006$; difference contrast, $t=-2.45, p<$ $0.05)$ and the poststimulus periods were lower than baseline. No side difference was detected. No significant differences were found between data obtained during the waiting and baseline 

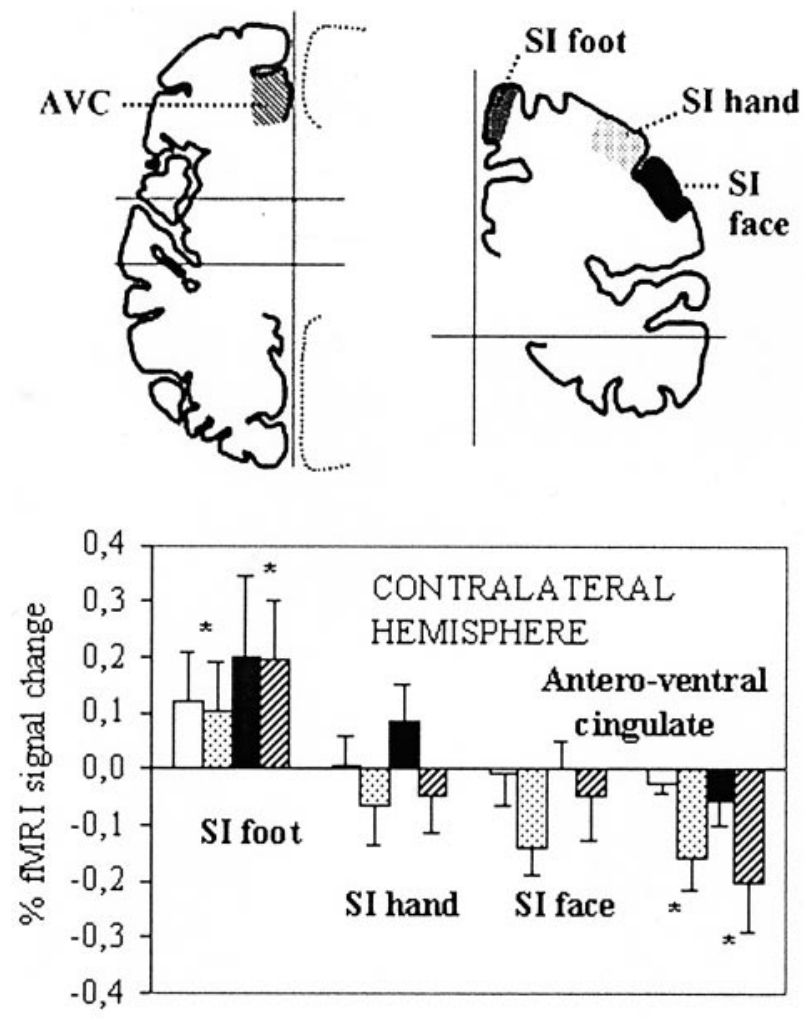

Waiting Post-Stimulus
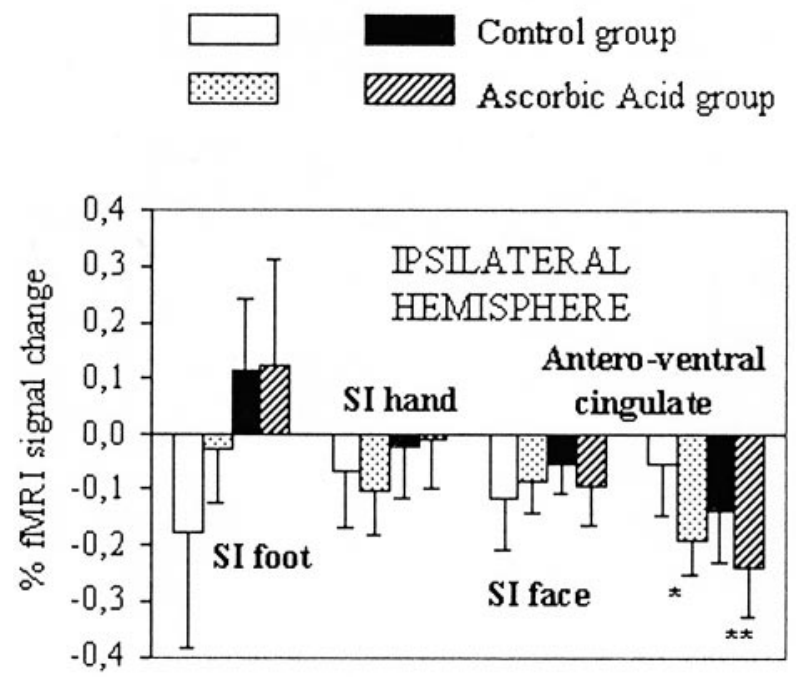

Figure 2. Experiment 1. Mean fMRI signal changes in selected ROIs. Top, Schematic drawings of the brain, showing the approximate location of ROIs in which significant mean fMRI signal changes occurred. The vertical and horizontal lines on the left correspond to the interhemispheric fissure and to the vertical planes passing through the anterior and posterior commissure, respectively; those on the right correspond to the interhemispheric fissure and to the horizontal plane passing through the anterior and posterior commissures. $A V C$, Anteroventral cingulate. Mean Talairach coordinates $(|x|, y, z$, expressed in millimeters) of the centers of mass of ROIs were as follows: $6,37,10$ for anteroventral cingulate; $9,-37,60$ for SI foot; 39, $-26,51$ for SI hand; 51, $-16,37$ for SI face. Data from the left and right hemispheres were pooled (see Materials and Methods), and therefore absolute (unsigned) values are given for the $x$ coordinate. For the $y$ coordinate, positive values are anterior, and negative values are posterior to the vertical plane passing through the anterior commissure. For the $z$ coordinate, positive values are superior to the horizontal plane passing through
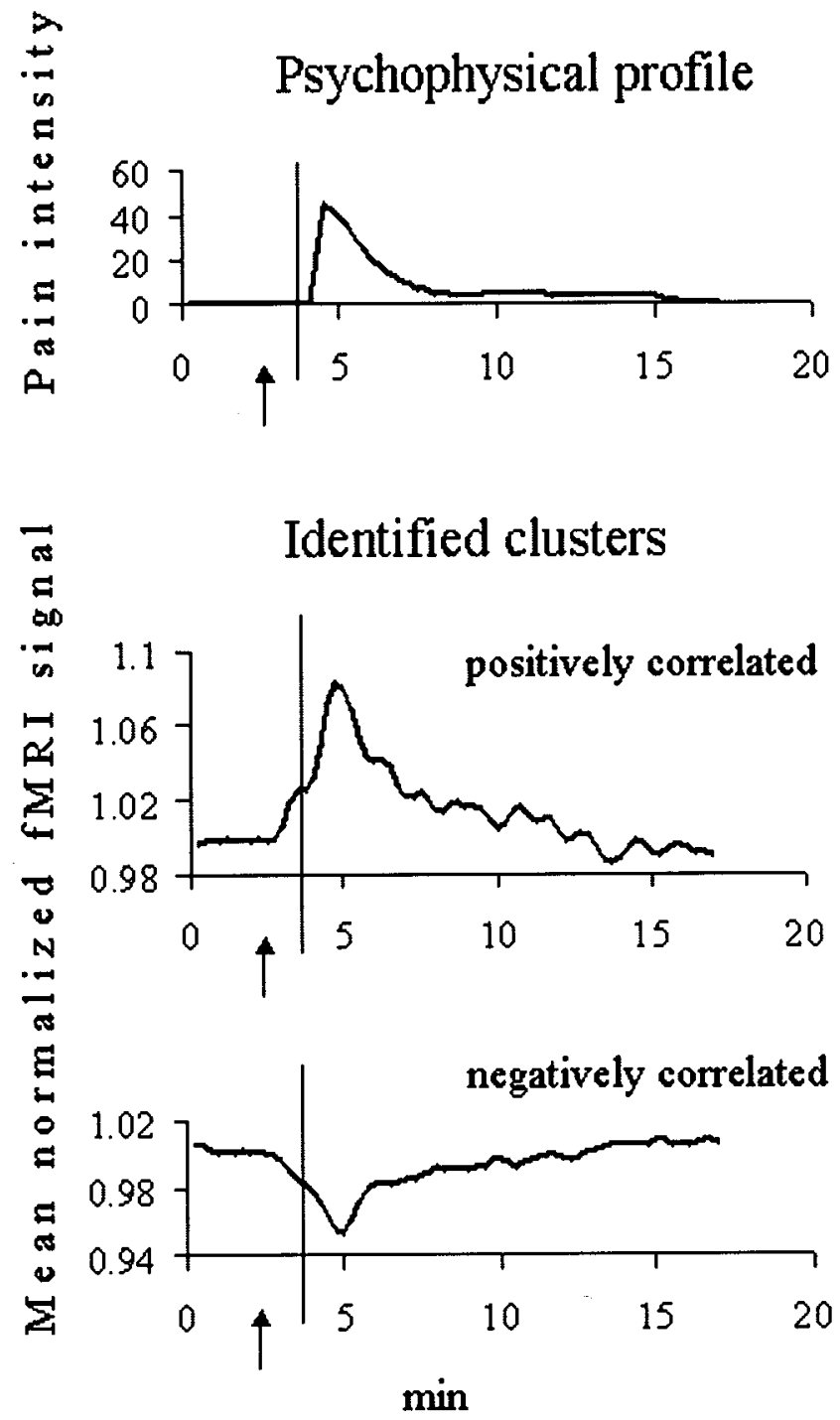

Figure 3. Signal changes in clusters encoding pain intensity over time in one representative subject. Time profiles of the perceived pain intensity (0-100 scale) (top) and of mean normalized fMRI signal intensities in cortical clusters whose signal time courses were positively (middle) or negatively (bottom) correlated with the individual psychophysical curve. Arrows point to the warning signal. Vertical lines indicate the time of the subcutaneous ascorbic acid injection. Note the changes from baseline activity before stimulation onset, which appear to be triggered by the warning signal.

periods in other cortical areas (data not shown). No significant effect of group, or of any interaction of group with other factors, was found.

fMRI signal changes in clusters correlated with the individual profiles of pain intensity

To specifically test whether signal changes during the waiting period involved cortical nociceptive networks, we examined the

the anterior and posterior commissures. Middle, Bottom, Mean normalized fMRI signal changes from baseline during the $45 \mathrm{sec}$ preceding stimulus onset (waiting period) and during the first minute after stimulus in the control and ascorbic acid groups. ${ }^{*} p<0.05$ and ${ }^{* *} p<0.01$ indicate data from the two groups significantly different from baseline (ANOVA plus difference contrast), respectively. 


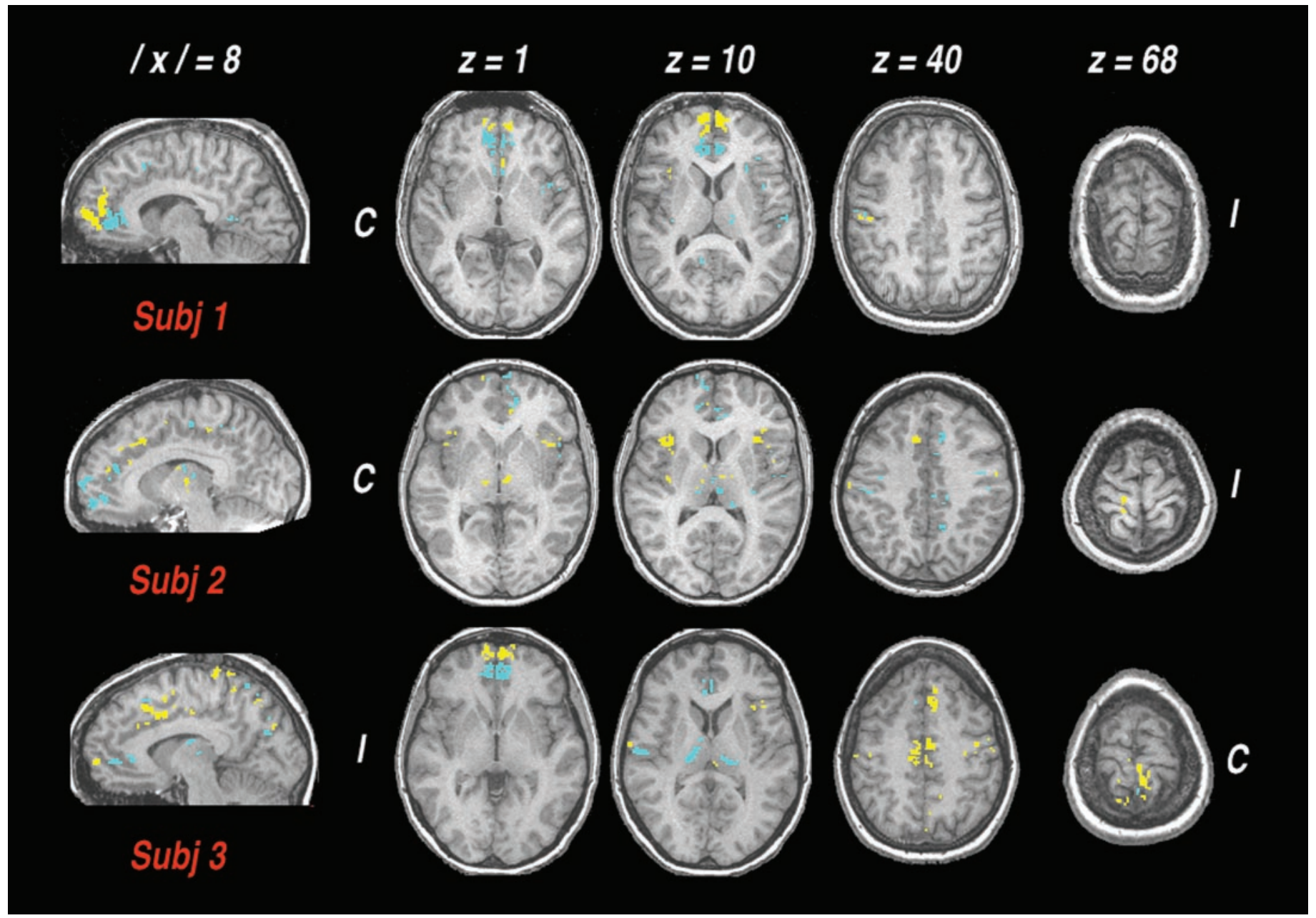

Figure 4. Spatial distribution of clusters showing significant anticipation- and pain-related responses, in three representative subjects. Functional maps are superimposed on Talairach-transformed axial images at the levels of medial prefrontal cortex and anterior insula $(z$ levels +1 and +10$)$, anterior cingulate $(z+40)$, and foot area of the primary somatosensory cortex $(z+68)$, and on a paramedian sagittal image on the hemisphere contralateral to the injected foot $(|x|=8)$. They depict the location of cortical clusters encoding pain intensity, which also showed significant signal changes during the anticipatory phase. Yellow and blue indicate clusters positively or negatively correlated with the individual psychophysical curve, respectively. $C$, $I$, Contralateral and ipsilateral to the injection side (axial images), respectively.

time profiles of activity in cortical clusters showing positive or negative correlations with the individual psychophysical pain intensity curve (Fig. 3). Such clusters were identified in 11 of 14 subjects of the ascorbic acid group. Three subjects experiencing very mild pain (peak intensity of 10) displayed no positively or negatively correlated clusters at all.

The spatial distribution of the identified clusters showed interindividual variations (Fig. 4). Positively correlated clusters were found predominantly in the contralateral SI foot area and bilaterally in the rostral anterior cingulate, medial prefrontal cortex, and anterior insula. Negatively correlated clusters were found bilaterally in the anteroventral cingulate. A few clusters were also found in the posterior insula/parietal operculum. For each ROI, the spatial location of clusters was similar in the two hemispheres (Table 1). Apart from SI, no significant difference was found between the spatial extent of the contralateral and the ipsilateral responses. In both hemispheres, the spatial extent of the identified clusters was linearly related to peak pain intensity (contralateral, Pearson's correlation coefficient, $r=0.789, p<0.001$; ipsilateral, $r=0.736, p<0.005)$. We also noted positively and negatively correlated clusters at the thalamic level in some subjects (Fig. 4).
Significant signal changes during the waiting period compared with baseline were present in all the identified clusters within the selected ROIs, although less intense than peak changes during the first 3 min of noxious input (on average, 38 and $28 \%$ as large for contralateral and ipsilateral positively correlated clusters, and 34 and $36 \%$ as large for contralateral and ipsilateral negatively correlated clusters, respectively) (Fig. 5).

\section{Experiment 2}

To test whether the mean fMRI signal increases in the contralateral foot representation area of SI during the waiting period were indeed related to anticipation, we performed a second experiment in which we compared fMRI signal changes after an identical innocuous somatosensory stimulus in two conditions: cue, when subjects were told that this signaled an impending, potentially noxious input, and control, when subjects knew that no additional stimulus would follow. In an open interview at the end of the experimental session, all subjects reported increased arousal during the waiting period in the cue condition but not during the corresponding period of the control condition. This was paralleled by an heart rate increase over baseline in the cue condition only (control, from $71 \pm 3$ to $71 \pm 3$ beats/min; paired 
Table 1. Spatial location of cortical clusters showing signal changes significantly related to the psychophysical pain intensity profile

\begin{tabular}{|c|c|c|c|c|c|c|c|c|}
\hline & \multicolumn{8}{|c|}{ Positively correlated clusters } \\
\hline & \multicolumn{4}{|c|}{ Contralateral hemisphere } & \multicolumn{4}{|c|}{ Ipsilateral hemisphere } \\
\hline & \multirow[b]{2}{*}{$n$} & \multicolumn{3}{|c|}{ Mean Talairach coordinates $(\mathrm{mm})$} & \multirow[b]{2}{*}{$n$} & \multicolumn{3}{|c|}{ Mean Talairach coordinates $(\mathrm{mm})$} \\
\hline & & $X$ & $Y$ & $z$ & & $x$ & $y$ & $z$ \\
\hline SI foot & 6 & $|10|$ & -35 & 65 & 1 & $|14|$ & -37 & 65 \\
\hline Anterior cingulate & 6 & $|7|$ & 19 & 34 & 6 & $|4|$ & 21 & 33 \\
\hline Anterior insula & 5 & $|35|$ & 14 & 7 & 7 & $|35|$ & 15 & 4 \\
\hline Medial prefrontal cortex & 8 & $|7|$ & 59 & 4 & 8 & $|6|$ & 56 & 0 \\
\hline
\end{tabular}

\begin{tabular}{|c|c|c|c|c|c|c|c|c|}
\hline & \multicolumn{8}{|c|}{ Negatively correlated clusters } \\
\hline & \multicolumn{4}{|c|}{ Contralateral hemisphere } & \multicolumn{4}{|c|}{ Ipsilateral hemisphere } \\
\hline & \multirow[b]{2}{*}{$n$} & \multicolumn{3}{|c|}{$\begin{array}{l}\text { Mean Talairach coordinates } \\
(\mathrm{mm})\end{array}$} & \multirow[b]{2}{*}{$n$} & \multicolumn{3}{|c|}{$\begin{array}{l}\text { Mean Talairach coordinates } \\
(\mathrm{mm})\end{array}$} \\
\hline & & $x$ & Y & $z$ & & $x$ & $y$ & 2 \\
\hline Anteroventral cingulate & 7 & $|8|$ & 40 & 6 & 7 & $|6|$ & 40 & 5 \\
\hline
\end{tabular}

$n$, Number of subjects.

\section{Identified clusters}

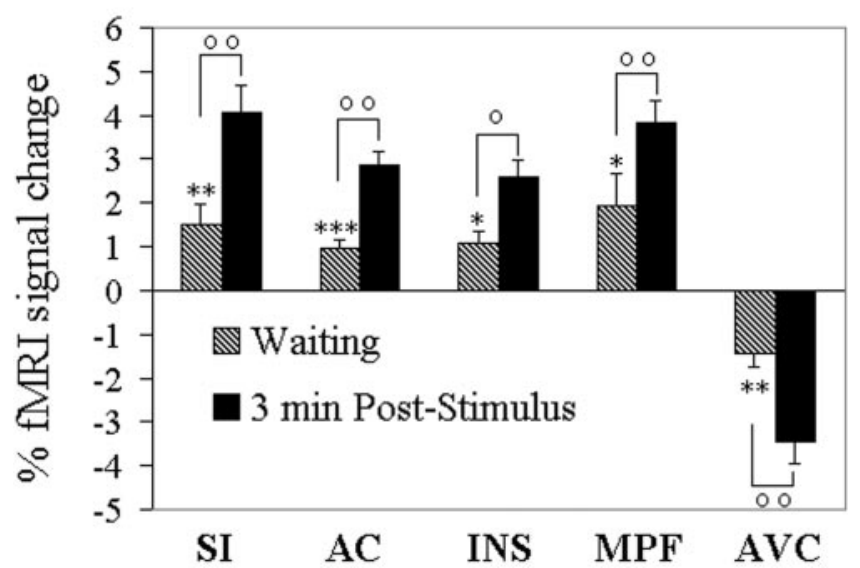

Figure 5. Mean normalized fMRI signal changes from baseline in clusters showing positive or negative correlations with the individual psychophysical profile during the waiting period and the first 3 min after stimulation. ${ }^{*} p<0.05,{ }^{*} p<0.01$, and ${ }^{* * *} p<0.001$ indicate different from baseline. ${ }^{\circ} p<0.05$ and ${ }^{\circ 0} p<0.01$ indicate different from the waiting period. All data from the poststimulus period were different from baseline at $p<0.001$. $A C$, Anterior cingulate; INS, anterior insula; $M P F$, medial prefrontal cortex. Other abbreviations as in text and Figure 2.

$t=0.40, \mathrm{NS}$; cue, from $72 \pm 3$ to $78 \pm 3$ beats $/ \mathrm{min}$; paired $t=$ $-9.93, p<0.001$ ) (Fig. 6).

ANOVA showed that mean fMRI signal intensity in the contralateral foot area of SI was differently modulated according to time and condition (time $\times$ condition $\times$ side, $F=9.08, p<0.05$ ). Values in the contralateral foot SI area were higher than ipsilateral ones in images acquired during the waiting period in the cue condition (paired $t=2.55, p<0.05$, one-tailed) when subjects expected a potentially noxious stimulation but not during the corresponding period in the control condition (paired $t=-0.94$, NS). No similar effects were found in the hand or face areas of SI (Fig. 7).

\section{DISCUSSION}

This study demonstrates changes of the activity of nociceptive networks in several cortical regions, likely involved in different aspects of pain (Bushnell et al., 1999; Casey, 1999; Ingvar and Hsieh, 1999; Treede et al., 1999, 2000), while subjects expected a potentially noxious somatic stimulation. Conceivably, this is attributable to the interplay of several processes, such as coping with the impending stimulus and sustained spatial attention.

\section{Anticipation-related changes of activity in the postcentral gyrus}

SI is mainly involved in the sensory-discriminative aspects of pain (Bushnell et al., 1999). Because increases in mean fMRI signals during anticipation were found only in the appropriate contralateral somatotopic area, they are likely to be related mainly to top-down (e.g., attentional) mechanisms enhancing activity of specific neuronal populations rather than to a diffuse arousal effect. Modulation by attention of ongoing stimulus-evoked activity has been described by electrophysiological experiments in the primary visual (Motter, 1993; Roelfsema et al., 1998) and somatosensory (Hyvarinen et al., 1980; Hsiao et al., 1993) cortex of the macaque monkey. Recent mapping studies reported similar effects in human primary sensory areas (Brefczynski and DeYoe, 1999; Burton et al., 1999; Somers et al., 1999). Notably, in the present study, increased signal was found in the absence of actual stimulation. Thus, our results support the hypothesis advanced by Roland (1981) of a somatotopic activation of SI during anticipation of innocuous somatosensory stimuli and are in line with a recent study showing increased fMRI signals in the putative SI foot area during anticipation of tickling (Carlsson et al., 2000).

As mentioned in the introductory remarks, previous studies failed to detect activation in SI during anticipation of pain (Drevets et al., 1995). Several methodological differences might explain these apparent discrepancies. Previous exposure to painful stimuli may trigger endogenous inhibitory systems limiting the transmission of noxious information to cortical areas and/or direction of attentional resources away from the stimulus (Hsieh et al., 1999). In our study, subjects had never experienced the noxious stimulus before. Thus, the results cannot be related to con- 


\section{Control}
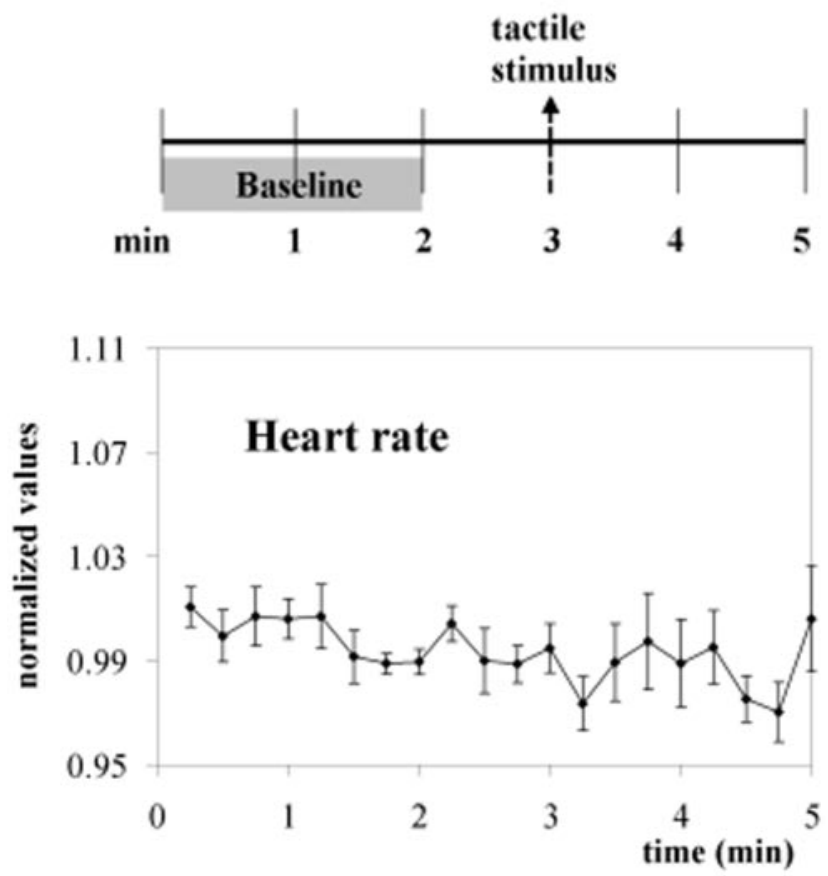

Cue
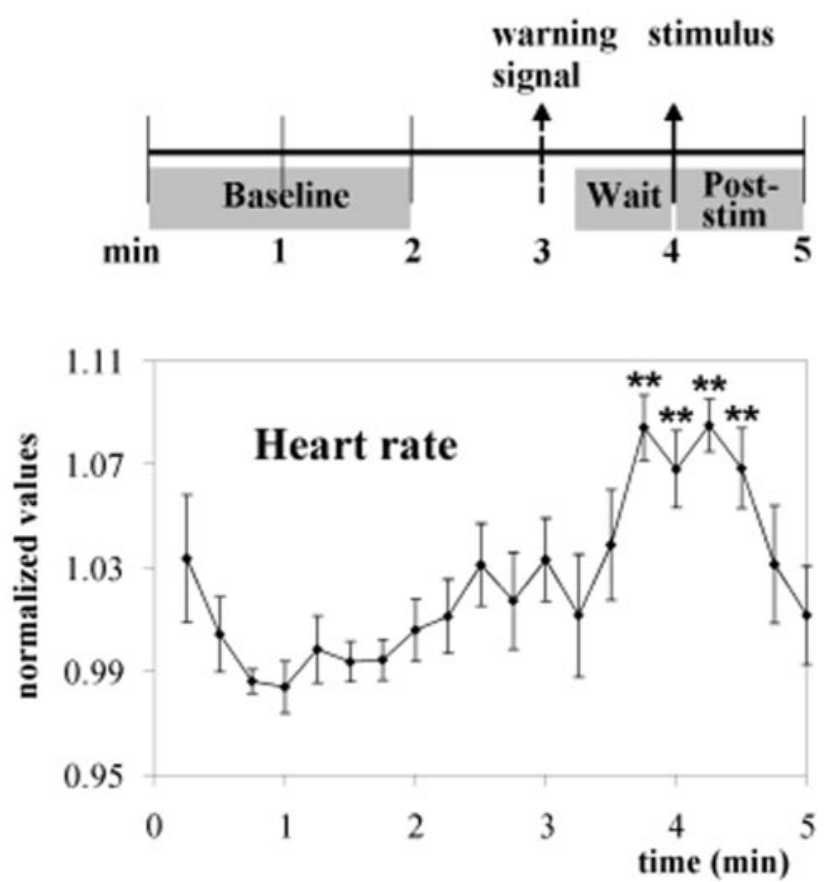

Figure 6. Experiment 2. Experimental design and mean heart rate data. Note the differences in the heart rate profiles between the control and cue conditions. ${ }^{* *} p<0.01$ indicates different from baseline; repeated-measures ANOVA plus simple contrast.

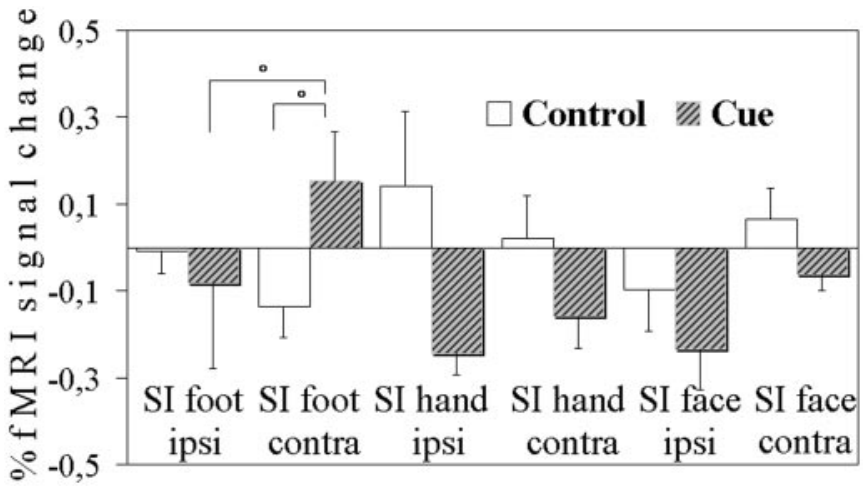

Figure 7. Experiment 2. Mean normalized fMRI signal changes from baseline during the waiting period in the cue condition and during the corresponding period in the control condition. ${ }^{\circ} p<0.05$ indicates significant differences.

ditioning or memory. Because increases in blood oxygenation level-dependent (BOLD) fMRI signals appear to be attributable mainly to synaptic activity (Logothetis et al., 2001), the observed mean fMRI signal increases in the SI foot region are likely attributable to a change in the baseline inputs to cortical neurons involved in processing signals from the attended body area. This "tonic" effect (Rees et al., 1997) may represent a general mechanism of top-down modulation of cortical sensory areas during spatial attention (Luck et al., 1997; Chawla et al., 1999; Kastner et al., 1999).

The decreased fMRI signals in the hand and face SI areas are in accordance with a previous PET study showing decreased flow in nonsomatotopically related portions of SI during anticipation of noxious input (Drevets et al., 1995) and fMRI findings of decreased signals in SI regions processing information from unattended parts of the body during tickling expectations (Carlsson et al., 2000). Decreased BOLD fMRI signals may be interpreted to reflect decreased local blood flow either as a consequence of changes of ongoing neuronal activity (for discussion, see Gusnard et al., 2001; Logothetis et al., 2001) or of a "stealing" mechanisms attributable to activation of neighbor regions. Because the hand and face SI regions are larger than, and not contiguous to, the SI foot area, we believe that the first interpretation is more likely to explain our results. Thus, we postulate the simultaneous engagement of two top-down cortical mechanisms shaping the activity of lateral thalamocortical somatosensory systems, enhancing and suppressing functional activity levels of different cortical populations to filter out irrelevant information (Drevets et al., 1995; Shulman et al., 1997; Ghatan et al., 1998). It remains to be established whether the same frontoparietal network that appears to be the "source" of attentional mechanisms in the visual modality (Corbetta, 1998; Coull, 1998) is also involved in modulation of the somatosensory, and specifically, nociceptive system.

\section{Changes of activity in other cortical areas}

Other cortical regions, such as the anterior insula, cingulate cortex, and medial prefrontal cortex, which are likely to participate in cognitive and emotional processing and vegetative control (Treede et al., 1999, 2000; Price, 2000), showed clusters encoding pain intensity over time and displaying activity changes during anticipation of noxious input. Previous PET or fMRI studies by several groups, including our own (Vogt et al., 1996; Davis et al., 1998; Porro et al., 1998; Baron et al., 1999), have shown considerable intersubject variability in the cortical response to noxious stimuli. We chose a rather conservative statistical threshold, and, therefore, we may have missed activations in some subjects and in some regions. However, cluster location is generally consistent 
with previous PET and fMRI findings, and a linear relationship was found between the spatial extent of the response in both hemispheres and peak pain intensity. These findings confirm the distributed, bilateral quality of the cortical network encoding pain intensity (Derbyshire et al., 1997; Porro et al., 1998; Coghill et al., 1999).

Although the specific role of the above mentioned areas in pain mechanisms is yet to be definitely understood, there is increasing evidence that each may contribute to pain experience. The anterior insular cortex is involved in multimodal integration and visceromotor control (Augustine, 1996) and is activated during pain in experimental animals (Porro et al., 1999) and in humans (Treede et al., 2000). The anterior cingulate cortex is involved in anticipation (Murtha et al., 1996), pain, and attention (Davis et al., 1997; Derbyshire et al., 1998; Peyron et al., 1999; Davis et al., 2000). The anteroventral cingulate was the only area to show mean fMRI signal decreases during anticipation apart from SI. It comprises different subareas with a complex pattern of connection with limbic structures and appears important for the integration of emotional and cognitive processes and vegetative activity (Damasio, 1994; Devinsky et al., 1995; Ongur and Price, 2000). Changes of mood state are indeed accompanied by metabolic and blood flow changes in this area under physiological and pathological conditions (George et al., 1995; Drevets et al., 1997). Blood flow decreases during anticipation of a noxious electrical stimulus were also found in a recent PET study and were inversely related to anxiety levels (Simpson et al., 2001). Decreases of blood flow in the medial prefrontal cortex are common to a variety of attentiondemanding tasks (Shulman et al., 1997). This may be attributed to the interruption of a "default" state of ongoing mental activity (Gusnard et al., 2001; Raichle et al., 2001) that, in the present study, could result at least in part from sustained attention directed toward the site in which noxious stimulation was expected.

Blood flow increases in more rostral aspects of the medial prefrontal cortex, close to the ones found here (likely corresponding to medial area 10), have been described previously by PET during anticipation of unpredictable pain (Hsieh et al., 1999). This area is likely to be involved in second-order appraisals of nociceptive input (Price, 2000) and prospective memory (Burgess et al., 2001), which might explain its activation during expectation of potentially noxious events.

\section{Significance of changes of the activity of cortical nociceptive circuits during anticipation of pain}

Previous behavioral studies showed that uncertainty about impending noxious stimulation modifies pain unpleasantness and decreases pain tolerance (Staub et al., 1971). To the best of our knowledge, we provide the first demonstration in humans of potential neural mechanisms for such effects, showing that cortical clusters encoding pain intensity, which are presumably involved in perceptual aspects of pain (Porro et al., 1998), undergo significant changes in each investigated area during anticipation of noxious input. This suggests that the activity of nociceptive networks may be directly affected by cognitive factors (Davis et al., 2000) or, at least, that most anticipation- and pain-related neurons show a close spatial overlap.

The present results are thus at variance with those of Ploghaus et al. (1999) who described separate foci in some cortical regions, such as the anterior cingulate and insula, which were activated during anticipation but not during acute thermal pain. Conversely, in the study by Ploghaus et al., cortical clusters activated during pain showed apparently no change during anticipation: the relationships between the activity of these clusters and psychophysical aspects of pain were not investigated. We have evidence from other data that some cortical clusters are indeed activated mainly during the anticipatory phase and are spatially segregated from the ones related to pain (C. A. Porro, V. Cettolo, M. P. Francescato, and P. Baraldi, unpublished observations). However, the present fMRI findings suggest a close link between anticipation and cortical circuits encoding pain intensity, thus confirming and extending electrophysiological data in nonhuman primates showing that anticipation of noxious input activates nociceptive neurons in the anterior cingulate cortex (Koyama et al., 1998). Interestingly, anticipation of pain appears to affect the whole cortical nociceptive network in humans.

Our study was not aimed to identify cortical systems responding to non-noxious somatosensory input, and, therefore, we cannot establish whether they were activated or inhibited during the anticipatory phase. Sawamoto et al. (2000) described recently modulation of cortical clusters responding to warm stimuli in a condition in which subjects did not know in advance whether a thermal stimulus would be painful or not. Additional studies are needed to assess the specificity of the anticipation-related effects in different experimental paradigms.

The possible clinical relevance of these findings deserves a final comment. The widespread "priming" effect of nociceptive circuits during anticipation emphasize the need for an appropriate psychological approach to predictable or potentially noxious events.

\section{REFERENCES}

Andersson LR, Lilja A, Hartvig P, Langstrom B, Gordh T, Handwerker H, Torebjork E (1997) Somatotopic organization along the central sulcus, for pain localization in humans, as revealed by positron emission tomography. Exp Brain Res 117:192-199.

Augustine JR (1996) Circuitry and functional aspects of the insular lobe in primates including humans. Brain Res Rev 22:229-244.

Bandettini PA, Jesmanowicz A, Wong EC, Hyde JS (1993) Processing strategies for time-course data sets in functional MRI of the human brain. Magn Reson Med 30:161-173.

Baron F, Baron Y, Disbrow E, Roberts TPL (1999) Brain processing of capsaicin-induced secondary hyperalgesia. Neurology 53:548-557.

Brefczynski J, DeYoe E (1999) A physiological correlate of the "spotlight" of visual attention. Nat Neurosci 2:370-374.

Burgess PW, Quayle A, Frith CD (2001) Brain regions involved in prospective memory as determined by positron emission tomography. Neuropsychologia 39:545-555.

Burton H, Abend S, MacLeod A, Sinclair R, Snyder A, Raichle M (1999) Tactile attention tasks enhance activation in somatosensory regions of parietal cortex: a positron emission tomography study. Cereb Cortex 9:662-674.

Bushnell M, Duncan G, Hofbauer R, Chen J-I, Carrier B (1999) Pain perception: is there a role for primary somatosensory cortex? Proc Nat Acad Sci USA 96:7705-7709.

Carlsson K, Petrovic P, Skare S, Petersson KM, Ingvar M (2000) Tickling expectations: neural processing in anticipation of a sensory stimulus. J Cogn Neurosci 12:691-703.

Casey K (1999). Forebrain mechanisms of nociception and pain: analysis through imaging. Proc Natl Acad Sci USA 96:7668-7674.

Chawla D, Rees G, Friston K (1999) The physiological basis of attentional modulation in extrastriate visual areas. Nat Neurosci 2:671-676

Chua P, Kraus M, Toni I, Passingham R, Dolan R (1999) A functional anatomy of anticipatory anxiety. NeuroImage 9:563-571.

Coghill R, Sang C, Maisog J, Iadarola M (1999) Pain intensity processing within the human brain: a bilateral, distributed mechanism. J Neurophysiol 82:1934-1943.

Corbetta M (1998) Frontoparietal cortical networks for directing attention and the eye to visual locations: identical, independent, or overlapping neural systems? Proc Natl Acad Sci USA 95:831-838.

Coull J (1998) Neural correlates of attention and arousal: insights from electrophysiology, functional neuroimaging and psychopharmacology. Prog Neurobiol 55:343-361

Cox R (1996) AFNI: software for analysis and visualization of functional magnetic resonance neuroimages. Comput Biomed Search 29:162-173.

Damasio A (1994) Descartes' error. New York: Putnam.

Davis KD, Taylor S, Crawley A, Wood M, Mikulis D (1997) Functional 
MRI of pain- and attention-related activation in the human cingulate cortex. J Neurophysiol 77:3370-3380.

Davis KD, Kwan CL, Crawley AP, Mikulis DJ (1998) Functional MRI study of thalamic and cortical activations evoked by cutaneous heat, cold, and tactile stimuli. J Neurophysiol 80:1533-1546.

Davis KD, Hutchison WD, Lozano AM, Tasker RR, Dostrovsky JO (2000) Human anterior cingulate cortex neurons modulated by attention-demanding tasks. J Neurophysiol 83:3575-3577.

Derbyshire S, Jones A, Gyulai F, Clark S, Townsend D, Firestone L (1997) Pain processing during three levels of noxious stimulation produces different patterns of central activity. Pain 73:431-445.

Derbyshire S, Vogt B, Jones A (1998) Pain and Stroop interference tasks activate separate processing modules in anterior cingulate cortex. Exp Brain Res 118:52-60.

Devinsky O, Morrell M, Vogt B (1995) Contributions of anterior cingulate cortex to behavior. Brain 118:279-306.

Drevets W, Burton H, Videen T, Snyder A, Simpson J, Raichle M (1995) Blood flow changes in human somatosensory cortex during anticipated stimulation. Nature 373:249-252.

Drevets W, Price J, Simpson J, Todd R, Reich T, Vannier M, Raichle M (1997) Subgenual prefrontal cortex abnormalities in mood disorders. Nature 386:824-827.

Gelnar PA, Krauss BR, Sheehe PR, Szevereny NM, Apkarian AV (1999) A comparative fMRI study of cortical representations for thermal painful, vibrotactile, and motor performance tasks. NeuroImage 10:460-482.

George M, Ketter T, Parekh P, Horwitz B (1995) Brain activity during transient sadness and happiness in healthy women. Am J Psychiatry 152:341-351.

Geyer S, Schleicher A, Zilles K (1999) Areas 3a, 3b and 1 of primary somatosensory cortices. NeuroImage 10:63-83.

Ghatan P, Hsieh J, Petersson K, Stone-Elander S, Ingvar M (1998) Coexistence of attention-based facilitation and inhibition in the human cortex. NeuroImage 7:23-29.

Greenspan JD, Lee RR, Lenz FA (1999) Pain sensitivity alterations as a function of lesion location in the parasylvian cortex. Pain 81:273-282.

Gusnard DA, Akbudak E, Shulman G, Raichle ME (2001) Medial prefrontal cortex and self-referential mental activity: relation to a default mode of brain function. Proc Natl Acad Sci USA 98:4259-4264.

Hofbauer R, Rainville P, Duncan G, Bushnell M (2001) Cortical representation of the sensory dimension of pain. J Neurophysiol 86:402-411.

Hsiao S, O'Shaugnessy D, Johnson K (1993) Effects of selective attention on spatial form processing in monkey primary and secondary somatosensory cortex. J Neurophysiol 70:444-447.

Hsieh J, Stone-Elander S, Ingvar M (1999) Anticipatory coping of pain expressed in the human anterior cingulate cortex: a positron emission tomography study. Neurosci Lett 262:61-64.

Hyvarinen J, Poranen A, Jokinen Y (1980) Influence of attentive behavior on neuronal responses to vibration in primary somatosensory cortex of the monkey. J Neurophysiol 43:870-882.

Ingvar M, Hsieh JC (1999) The image of pain. In: Textbook of pain (Wall PD, Melzack M, eds), pp 215-233. Edinburgh: Churchill Livingstone.

Kastner S, Pinsk M, De Weerd P, Desimone R, Ungerleider L (1999) Increased activity in human visual cortex during directed attention in the absence of visual stimulation. Neuron 22:751-761.

Koyama T, Tanaka Y, Mikami A (1998) Nociceptive neurons in the macaque anterior cingulate cortex activate during anticipation of pain. NeuroReport 9:2663-2667.

Logothetis NK, Pauls J, Augath M, Trinath T, Oeltermann A (2001) Neurophysiological investigation of the basis of the fMRI signal. Nature 412:150-157.

Luck S, Chelazzi L, Hillyard S, Desimone R (1997) Neural mechanisms of spatial selective attention in areas V1,V2, and V4 of macaque visual cortex. J Neurophysiol 77:24-42.

Motter B (1993) Focal attention produces spatially selective processing in visual cortical areas V1, V2 and V4 in the presence of competing stimuli. J Neurophysiol 70:909-919.

Murtha S, Chertkow H, Beauregard M, Dixon R, Evans A (1996) Anticipation causes increased blood flow to the anterior cingulate cortex. Hum Brain Mapp 4:103-112.

Ongur D, Price JL (2000) The organization of networks within the orbital and medial prefrontal cortex of rats, monkeys and humans. Cereb Cortex 10:206-219.

Ono M, Kubik S, Abernathey C (1990) Atlas of the cerebral sulci. Stuttgart, Germany: Thieme.

Petrovic P, Petersson K, Ghatan P, Stone-Elander S, Ingvar M (2000)
Pain-related cerebral activation is altered by a distracting cognitive task. Pain 85:19-30.

Peyron R, Garcia-Larrea L, Gregoire M-C, Costes N, Convers P, Lavenne F, Mauguiere F, Michel D, Laurent B (1999) Haemodynamic brain responses to acute pain in humans. Sensory and attentional networks. Brain 122:1765-1779.

Ploghaus A, Tracey I, Gati J, Clare S, Menon R, Matthews P, Rawlins J (1999) Dissociating pain from its anticipation in the human brain. Science 284:1979-1981.

Porro CA, Cavazzuti M (1996) Functional imaging studies of the pain system in man and animals. Prog Brain Res 110:47-62.

Porro CA, Cettolo V, Francescato M, Baraldi P (1998) Temporal and intensity coding of pain in human cortex. J Neurophysiol 80:3312-3320.

Porro CA, Cavazzuti M, Baraldi P, Giuliani D, Panerai AE, Corazza R (1999) Central nervous system pattern of metabolic activity during tonic pain: evidence for modulation by beta-endorphin. Eur J Neurosci 11:874-888.

Price DD (1999) Psychological mechanisms of pain and analgesia. Seattle: IASP.

Price DD (2000) Psychological and neural mechanisms of the affective dimension of pain. Science 288:1769-1772.

Rademacher J, Caviness VS, Steinmetz H, Galaburda AM (1993) Topographical variation of the human primary cortices: implications for neuroimaging, brain mapping, and neurobiology. Cereb Cortex 3:313-329.

Raichle ME, MacLeod AM, Snyder AZ, Powers WJ, Gusnard DA, Shulman G (2001) A default mode of brain function. Proc Natl Acad Sci USA 98:676-682.

Rainville P, Duncan G, Price D, Carrier B, Bushnell M (1997) Pain affect encoded in human anterior cingulate but not somatosensory cortex. Science 277:968-971.

Rees G, Frackowiak R, Frith C (1997) Two modulatory effects of attention that mediate object categorization in human cortex. Science 275:835-838.

Roelfsema P, Lamme V, Spekreijse H (1998) Object-based attention in primary visual cortex of the macaque monkey. Nature 395:376-381.

Roland PE (1981) Somatotopical tuning of postcentral gyrus during focal attention in man. A regional cerebral blood flow study. J Neurophysiol 46:744-754.

Rossi A, Decchi B (1997) Changes in Ib heteronymous inhibition to soleus motoneurones during cutaneous and muscle nociceptive stimulation in humans. Brain Res 774:55-61.

Rumeau C, Tzourio N, Peretti-Vitton P, Levrier O, Joliot M, Mazoyer B, Salamon G (1994) Location of hand function in the sensorimoto cortex: MR and functional correlation. Am J Neuroradiol 15:567-572.

Sawamoto N, Honda M, Okada T, Hanakawa T, Kanda M, Fukuyama H, Konishi J, Shibasaki H (2000) Expectation of pain enhances responses to nonpainful somatosensory stimulation in the anterior cingulate cortex and parietal operculum/posterior insula: an event-related functional magnetic resonance imaging study. J Neurosci 20:7438-7445.

Shulman G, Corbetta M, Buckner R, Raichle M, Fiez J, Miezin F, Petersen S (1997) Top-down modulation of early sensory cortex. Cereb Cortex 7:193-206.

Simpson JR, Drevets WC, Snyder AZ, Gusnard DA, Raichle ME (2001) Emotion-induced changes in human medial prefrontal cortex. II. During anticipatory anxiety. Proc Natl Acad Sci USA 98:688-693.

Somers D, Dale A, Seiffert A, Tootell R (1999) Functional MRI reveals spatially specific attentional modulation in human primary visual cortex. Proc Natl Acad Sci USA 96:1663-1668.

Staub E, Tursky B, Schwartz GE (1971) Self-control and predictability: their effects on reactions to aversive stimulation. J Pers Soc Psychol 18:157-162.

Talairach J, Tournoux P (1988) Co-planar stereotaxic atlas of the human brain. Stuttgart, Germany: Thieme.

Treede RD, Kenshalo D, Gracely R, Jones AKP (1999) The cortical representation of pain. Pain 79:105-111.

Treede RD, Apkarian AV, Bromm B, Greenspan JD, Lenz FA (2000) Cortical representation of pain: functional characterization of nociceptive areas near the lateral sulcus. Pain 87:113-119.

Vogt BA, Nimchinsky EA, Vogt LJ, Hof PR (1995) Human cingulate cortex: surface features, flat maps, and cytoarchitecture. J Comp Neurol 359:490-506.

Vogt BA, Derbyshire S, Jones AKP (1996) Pain processing in 4 regions of human cingulate cortex localized with coregistered PET and MR imaging. Eur J Neurosci 8:1461-1473.

Yousry TA, Schmid UD, Alkadhi H, Schmidt D, Peraud A, Buettner A, Winkler P (1997) Localization of the motor hand area to a knob on the precentral gyrus: a new landmark. Brain 120:141-157. 